\title{
hAMSC-CM Attenuates Paraquat-Induced A549 Cell Damage by Reducing Endoplasmic Reticulum Stress and Oxidative Stress
}

\section{Futuan Liao}

Affiliated Hospital of Zunyi Medical University

\section{Liming Gong}

Affiliated Hospital of Zunyi Medical University

Lijing Jia

301 Military Hospital: Chinese PLA General Hospital

Jianhong Wang

Affiliated Hospital of Zunyi Medical University

Tongying Liu

Affiliated Hospital of Zunyi Medical University

\section{Tingting Huang}

Affiliated Hospital of Zunyi Medical University

Jing Liang

Affiliated Hospital of Zunyi Medical University

manhong zhou ( $\square$ manahongzhou@126.com )

Affiliated Hospital of Zunyi Medical College https://orcid.org/0000-0002-0824-1787

\section{Research}

Keywords: paraquat, A549 cells, amnion-derived mesenchymal stem cells, apoptosis, endoplasmic reticulum stress

Posted Date: August 19th, 2021

DOI: https://doi.org/10.21203/rs.3.rs-786419/v1

License: (c) (i) This work is licensed under a Creative Commons Attribution 4.0 International License.

Read Full License 


\section{Abstract}

Acute paraquat $(\mathrm{PQ})$ poisoning results in severe acute lung injury and pulmonary fibrosis, and there is no specific antidote; thus, the mortality rate of $\mathrm{PQ}$ poisoning is extremely high. The mechanism of poisoning may be associated with endoplasmic reticulum stress, oxidative stress damage and organ/tissue inflammation. Recent studies have reported that human amnion-derived mesenchymal stem cells (hAMSCs) secrete a variety of cytokines, and that hAMSC-conditioned medium (CM) has antiinflammatory and immunomodulatory effects. The aim of the present study was to investigate whether hAMSC-CM exerts protective effects against PQ toxicity in A549 cells. The data demonstrated that the activity of $A 549$ cells was decreased after $24 \mathrm{~h}$ of PQ exposure and that the cell viability of the hAMSC$\mathrm{CM}$ intervention group was higher compared with the PQ-only group. hAMSC-CM intervention decreased cell damage, apoptosis rates, oxidative stress indexes, $\mathrm{Bax} / \mathrm{Bcl}-2$ ratios and $\mathrm{CHOP}$ expression levels in poisoned cells by CCK-8 experiment, apoptosis detection, ROS content detection, and Western blot analysis respectively. In conclusion, hAMSC-CM may attenuate the cell damage caused by PQ by reducing endoplasmic reticulum stress and oxidative stress.

\section{Introduction}

In total, 250,000-370,000 people die from pesticide poisoning worldwide each year, particularly in less developed countries and regions (1). Paraquat (1,1-dichloro-4,4-bipyridine; PQ), which has been produced since 1962, has become one of the most commonly used herbicides in developing countries due to its low price, good herbicidal effects and availability. PQ can enter the body through the digestive tract, skin, and respiratory tract resulting in poisoning. In clinical practice, oral intake is the most common route of exposure. Previous studies have confirmed that there is a specific polyamine transporter system (PTS) on the membranes of alveolar epithelial cells (AECs)(2). After acute PQ poisoning, the PTS present on the alveolar cell membrane can specifically and actively transport PQ from the blood into alveolar cells, thus causing $P Q$ to concentrate in the lungs and subsequently damage $A E C s$ and other cells. Therefore, the lungs represent the primary target organs after $P Q$ poisoning (3). Acute $P Q$ poisoning is often serious, and there is still no specific detoxification drug for the treatment of $P Q$ poisoning; thus, the mortality rate is high, and it is essential to further explore methods to treat PQ poisoning(4). Mesenchymal stem cells (MSCs) are a class of adult stem cells with strong abilities to proliferate and differentiate (5). Previous studies have demonstrated that bone marrow and cord blood-derived MSCs selectively undergo chemotaxis, colonize lung injury sites, repair damaged alveolar cells and tissues directly or via the factor$1 /$ chemokine receptor CXCR4 matrix signaling pathway, exert paracrine effects and produce a variety of cytokines that exert protective effects against lung injury $(6,7)$. Human amnion-derived mesenchymal stem cells (hAMSCs) exert similar effects and have the advantages of easy and non-invasive acquisition, relatively few ethical concerns, low immunogenicity and strong amplification ability. hAMSCs can directly repair cellular and tissue damage. These beneficial therapeutic effects may originate from the hAMSCs themselves, possibly due to the various soluble factors produced by hAMSCs. hAMSCs can exert various therapeutic effects by releasing a variety of cytokines (8-13). In the present study, the aim was to 
investigate whether the soluble cytokines produced by hAMSCs and present in hAMSC-conditioned medium (hAMSC-CM) exert protective effects on human alveolar type Il-like epithelial cells (A549 cells) against damage caused by acute $P Q$ poisoning, and to perform preliminary studies to elucidate the underlying mechanisms.

\section{Materials And Methods}

Isolation and extraction of hAMSCs. Human fetal placentas were obtained from the Department of Obstetrics and Gynecology at the Affiliated Hospital of Zunyi Medical University (Zunyi, China). The present study was approved by the Ethics Committee of the Affiliated Hospital of Zunyi Medical University, and all volunteers signed informed consent form before participating. The human amniotic membrane is a thin film composed of a single layer of interconnected epithelial cells that contains human amnion epithelial stem cells (hAESCs) and hAMSCs (14). After amniotic membrane separation, the tissue samples were washed with PBS three times and the tissue was shredded by mechanical methods. Next, $0.25 \%$ trypsin-EDTA was added to a final concentration of $0.05 \mathrm{mg} / \mathrm{ml}$, and the tissues were digested by shaking at $37^{\circ} \mathrm{C}$ for $30 \mathrm{~min}$ before being filtered with a 300-mesh cell strainer. Then, the tissue in the strainer was transferred to a $50 \mathrm{ml}$ centrifuge tube and digested with collagenase IV at $37^{\circ} \mathrm{C}$ for $2 \mathrm{~h}$ until tissue lysis was complete. The samples were then rinsed with PBS and filtered, and the filtrate was collected and centrifuged at $790 \times \mathrm{g}(2500 \mathrm{rpm})$ for $10 \mathrm{~min}$ at $4^{\circ} \mathrm{C}$. The supernatant was discarded, and original hAMSC medium was added. The cells were mixed and seeded in $25 \mathrm{~cm} 2$ cell culture flasks at a density of $1 \times 106$ cells/ml for later use after cell counting.

Culture of cell lines and collection of hAMSC-CM. A549 human alveolar type II-like epithelial cells from Beina Chuanglian Biotechnology Co., Ltd (Beijing, China) and hAMSCs (between passages 3 and 5) were seeded at a density of $2 \times 106$ cells/ml (determined with a fully automatic multifunction microplate reader; Thermo Fisher Scientific, Inc.) in an incubator $\left(37^{\circ} \mathrm{C}, 5 \% \mathrm{CO}\right)$ and cultured in DMEM/low-glucose medium (HyClone; GE Healthcare Life Sciences) containing 10\% fetal bovine serum (FBS; Gemini Bio Products). The medium was changed once every two days. When the cells reached $80 \%$ confluence, they were subjected to $1: 2$ passaging. When the hAMSCs covered $80-90 \%$ of the culture flask, they were washed with PBS twice, and the medium was replaced with $5 \mathrm{ml}$ of DMEM/low- glucose medium containing $10 \% \mathrm{FBS}$. After additional culture for $24 \mathrm{~h}$ at $37^{\circ} \mathrm{C}$, the culture supernatant was aspirated, filtered through a $0.22 \mu \mathrm{m}$ filter, dispensed into an EP tube and stored at $-80^{\circ} \mathrm{C}$.

Experimental protocol. A549 cells were cultured in 96-well plates, treated with PQ to establish the model (Sigma-Aldrich; Merck KGaA) and immediately treated with hAMSC-CM for $24 \mathrm{~h}$ before Cell Counting Kit-8 (CCK-8) detection. A549 cells with appropriate PQ and hAMSC-CM concentrations were selected for intervention experiments and were divided into the following groups: i) control group; ii) PQ group (exposed to $P Q$ only); iii) $P Q+C M$ group (treated first with $P Q$ and then with hAMSC-CM); and iv) CM group (the hAMSC-CM control group). A549 cells were also cultured in 6-well plates, and apoptosis was measured by flow cytometry $24 \mathrm{~h}$ after treatment. Other A549 cells were cultured in a $25 \mathrm{~cm} 2$ cell culture flask. At $24 \mathrm{~h}$ after intervention, the morphology of the cells was observed, and the levels of ROS were 
detected. The expression levels of related proteins in the cells were detected by western blot analysis. The cytokine content in the supernatant was detected by ELISA.

CCK-8 experiment. A549 cells were cultured and then exposed to different concentrations of PQ solution $(0,250,500,750$ and $1,000 \mu \mathrm{M})$ for $24 \mathrm{~h}$ at $37^{\circ} \mathrm{C}$ ( 6 replicate wells per group). Then, $10 \mu$ of CCK- 8 reagent (Beijing Soleil Technology Co., Ltd.) was added to each well, and the cells were incubated at $37^{\circ} \mathrm{C}$ for $2 \mathrm{~h}$. The absorbance value at a wavelength of $450 \mathrm{~nm}$ was measured, and cell proliferation activity was calculated according to the following formula: cell proliferation activity $(\%)=($ experimental group OD value - zero well OD value) / (control group OD value - zero well OD value). Analyses were conducted in the same manner after hAMSC-CM was administered at concentrations of 25,50 and $75 \%$ volume fraction (ie, medium volume/total volume). The order of addition was medium, PQ solution and hAMSC$\mathrm{CM}$, and the cells were analyzed after $24 \mathrm{~h}$ of culture at $37^{\circ} \mathrm{C}$.

Qualitative observation of intracellular ROS. 2,7-Dichlorofluorescein diacetate (DCFH-DA) itself has no fluorescence and is hydrolyzed into DCFH by esterase after entering cells. In the presence of active oxygen, DCFH is oxidized to form a substance with strong green fluorescence, DCF, that cannot penetrate the cell membrane (15). The fluorescence of DCF has an excitation wavelength of $502 \mathrm{~nm}$, the emission peaks near $530 \mathrm{~nm}$ and the intensity is proportional to the level of intracellular ROS. Reactive oxygen species Assay Kit was obtained from Nanjing Jiancheng Bioengineering Institute. (catalog number: E0041-1and was used according to the manufacturer's protocol. After $24 \mathrm{~h}$ of culture at $37^{\circ} \mathrm{C}$, the cells in each group were washed once with PBS. Next, $40 \mu \mathrm{M}$ DCFH-DA dye was added to each group, and the mixture was incubated for $30 \mathrm{~min}$ at $37^{\circ} \mathrm{C}$. The development of fluorescence in the cells of each group was observed under a green light source with a fluorescence microscope (Olympus Corporation),100x.

Observation of cell morphology. After cells were cultured for $24 \mathrm{~h}$, cell morphology was observed under an inverted phase-contrast microscope (OLYMPUS, 200x). The cells in each group were washed twice with PBS, digested and centrifuged $(2,200 \times \mathrm{g} ; 5 \mathrm{~min})$ at $25^{\circ} \mathrm{C}$. The supernatant was discarded. The cells in the pellets were fixed with $1 \%$ citric acid for $24 \mathrm{~h}$ at $4^{\circ} \mathrm{C}$, dehydrated with different concentrations of ethanol and acetone, soaked, embedded, trimmed, sectioned and stained (stains: Uranyl Acetate and Lead Citrate were purchased from HEAD (BeiJing) Biotechology Co. Ltd). A transmission electron microscope (Zeiss, Germany, bar $=2 \mu \mathrm{m}$ ) was used to observe changes in tissue structure.

\section{Detection of oxidation index values and inflammatory factor levels in cells and their supernatants.}

Samples were prepared according to the corresponding kit instructions. The activity of superoxide dismutase (SOD) in A549 cells was determined using the xanthine oxidase method (Superoxide Dismutase assay kit, Nanjing Jiancheng Bioengineering Institute). The MDA content in A549 cells was detected by the thiobarbituric acid method (Cell Malondialdehyde assay kit, Nanjing Jiancheng Bioengineering Institute). The levels of interleukin (IL)-1 $\beta$, IL-6 and IL-10 in the supernatants of A549 cells were detected by ELISA (Wuhan Sanying Biotechnology). Blank control wells and standard wells were included in the experiment. The absorbance was measured at a wavelength of $450 \mathrm{~nm}$ to prepare a standard curve, and the concentrations of the samples to be tested were calculated. 
Apoptosis detection with Annexin V-FITC/propidium iodide (PI). Annexin V-FITC (TransDtetce Annexin VFITC/PI Cell Apoptosis Detection Kit) was purchased from TransGen Biotech (), and it can specifically bind to phosphatidylserine, which translocates to the membrane during apoptosis, and emit green fluorescence under excitation by blue light to enable apoptotic cells and normal cells to be distinguished. $\mathrm{PI}$ is a nucleic acid dye that cannot penetrate intact cell membranes; however, it can penetrate damaged cell membranes (including those of late apoptotic and dead cells) and stain the nuclei red. The signals of these two labels can be compared to distinguish between early and late apoptotic cells. After $24 \mathrm{~h}$ of culture, cells (density $2 \times 106$ )were digested with $0.25 \%$ trypsin without EDTA for 1 min and then collected, washed twice with cold PBS, and resuspended in binding buffer. Annexin V-FITC and PI were added to the cell suspensions, and the solutions were mixed and protected from light for $5 \mathrm{~min}$. Flow cytometry (BD, FACSCanto $^{\text {TM }}$ II Flow Cytometry System) was performed within $1 \mathrm{~h}$. BD FACSDiva Software (v8.0.2) was used for analysis. Quantification of the respective flow plots. Q1, Q2, Q3 and Q4 represent necrotic cells, late apoptotic cells, early apoptotic cells and normal cells, respectively.

Western blot detection of related protein expression. A549 cells of each group were collected, appropriate amounts of lysis buffer were added, and the mixtures were centrifuged at $4^{\circ} \mathrm{C}(39,588 \times \mathrm{g}, 10 \mathrm{~min}$; Beckman Coulter, Inc.). The supernatants were collected, and the protein concentrations were determined by the BCA method. Loading buffer was then added, and the proteins were denatured at $100^{\circ} \mathrm{C}$ for $5 \mathrm{~min}$. A total of $20 \mu \mathrm{g}$ of each protein sample was added to each lane for SDS-PAGE (separation gel concentration $12 \%$ and concentrated gel $5 \%$ ). After electrophoresis, the proteins were transferred to a PVDF microporous membrane at $200 \mathrm{~mA}$ for 70 min and blocked with 3\% BSA (Beijing Solarbio Science \& Technology Co., Ltd)in TBST (5\% of Tween 20) blocking solution for $2 \mathrm{~h}$ at room temperature. Then, the membrane was incubated with diluted primary antibodies, including a b-actin monoclonal antibody (cat. no. 66009-1-Ig; dilution 1:20,000), a Bcl-2-related X protein (Bax) polyclonal antibody (cat. no. 50599-2-Ig; dilution 1:7,000), a Bcl-2 polyclonal antibody (cat. no. 12789-1-AP, dilutions 1:3,000), and a CHOP polyclonal antibody (cat. no. 15204-1-AP, dilution 1:1,000) (Wuhan Sanying Biology Technology Co., Ltd.), overnight at $4^{\circ} \mathrm{C}$ and washed three times with TBST (15 min each time). The membrane was then incubated with secondary antibodies, including HRP-conjugated Affinipure goat anti-rabbit lgG $(\mathrm{H}+\mathrm{L})$ (dilution 1:5,000; Wuhan Sanying Biology Technology Co., Ltd.) and HRP-conjugated Affinipure goat antimouse IgG $(\mathrm{H}+\mathrm{L})$ (dilution 1:5,000; Wuhan Sanying Biology Technology Co., Ltd.) for $2 \mathrm{~h}$ at room temperature before being washed three times with TBST. A mixture of luminescent liquids $A$ and $B$ was evenly dropped onto the membrane $\mathbb{E} C \mathrm{CL}$ ENhanced Kit, Beijing Dining Biotechnology Co., Ltd囚, which was then placed in a Bio-Rad gel imaging system for imaging. The Western blot results were analyzed by grayscale scanning (Bio-Rad Laboratories, Inc.) using ImageJ software (National Institutes of Health\v5.2).

Statistical analysis. Experimental and measurement data are expressed as the mean \pm standard deviation $(\overline{\mathrm{x}} \pm \mathrm{s}$ ). SPSS 18.0 statistical software (SPSS, Inc.) was used for statistical analysis. One-way ANOVA was conducted among groups, the post hoc test used following one way ANOVA was Tukey's HSD, and $\mathrm{P}<$ 0.05 was considered to indicate statistical significance. 


\section{Results}

hAMSC-CM alleviates the cytotoxicity induced by PQ. In order to identify the dose of PQ, A549 cells were exposed to $\mathrm{PQ}$ at concentrations of $250,500,750$ and $1,000 \mu \mathrm{M}$ for $24 \mathrm{~h}$ and cell proliferation activity was decreased (Fig. 1A). In addition, decreased cell viability was observed after PQ exposure for more than $24 \mathrm{~h}$. The half-maximal inhibitory concentration (IC50) of PQ was calculated to be $816 \mu \mathrm{M}$, which was closest to $750 \mu \mathrm{M}$; therefore, $750 \mu \mathrm{M} \mathrm{PQ}$ was selected as the modeling concentration for subsequent experiments.

The hAMSC-CM group was subdivided into groups treated with volume fractions of 25,50 and $75 \%$ hAMSC-CM. The concentration of CCK-8 reagent was detected after $24 \mathrm{~h}$ of exposure or intervention in order to evaluate the toxicity of $\mathrm{PQ}$ to $\mathrm{A} 549$ cells. There were no differences in cell proliferation activity between the hAMSC-CM groups (with different concentrations of hAMSC-CM) and the control group. Cell proliferation was detected after the addition of different concentrations of hAMSC-CM. The proliferation of cells in the $P Q+C M$ group was higher than that in the $P Q$ group at all concentrations. Among the different concentrations of hAMSC-CM, the $50 \%$ volume fraction resulted in the highest proliferation rate of A549 cells (Fig. 1B).

hAMSC-CM alleviates PQ-induced oxidative stress and increases in inflammatory mediator levels hAMSCCM reduces the production of ROS in poisoned cells. Intracellular ROS content was measured with a DCFH-DA probe. The green fluorescence and ROS content of the control group and the CM group were lower than those of the $P Q$ group and the $P Q+C M$ group; however, the $P Q+C M$ group had less fluorescence and a lower ROS content than the PQ group. At $24 \mathrm{~h}$ after exposure to $\mathrm{PQ}$ at $750 \mu \mathrm{M}$ or intervention (with the $50 \%$ concentration of hAMSC-CM), DCFH-DA was added, and fluorescence microscopy was used to observe green fluorescence (Fig. 2A).

hAMSC-CM alleviates tissue damage in poisoned cells. The morphology of the cells was observed under an inverted microscope. The cells in the control group and the CM group were uniform under the inverted microscope, showing cobblestone-like morphology, good adherence and tight connections. After $24 \mathrm{~h}$ of $P Q$ exposure, the morphology, connections and adherence of the cells were altered; the cells were not tightly connected, the number of dead cells was increased and the amount of cell debris at the bottom of the culture flask was increased. The PQ + CM group had a greater cell density, more adherent cells and fewer dead cells than the PQ group (Fig. 2B).

The ultrastructural morphology of the cells in the control group and the CM group was observed via transmission electron microscopy. The cell size was uniform, the cell membranes were intact, microvilli were observed on the cell surface, the cytoplasm was uniform, various organelles and the nuclear membrane were intact. After PQ exposure, the numbers of microvilli and protrusions on the surfaces of A549 cells were reduced, and cell membrane budding, apoptotic bodies, swelling of the endoplasmic reticulum, increased number of autophagosomes, decreased numbers of organelles (such as mitochondria), abnormal nuclear chromatin distribution, and damage to organelles (such as the 
endoplasmic reticulum) were all observed in the $P Q+C M$ group. The changes were milder in the $P Q+C M$ group than in the $\mathrm{PQ}$ group (Fig. 2C).

hAMSC-CM reduces PQ-induced cellular oxidation. The MDA content in each group was detected with an MDA kit. The MDA content in the PQ group was higher than that in the control group, while the MDA content in the $P Q+C M$ group was lower than that in the $P Q$ group (Fig. 2D). The SOD content in each group was also detected. The SOD content in the PQ group was lower than that in the control group, while the SOD content in the $P Q+C M$ group was significantly higher than that in the PQ group (Fig. 2E). There were no significant differences in the levels of MDA and SOD between the CM group and the control group.

hAMSC-CM reduces PQ-induced increases in inflammatory cytokine levels. The levels of certain ILs in the supernatant in each group was detected using ELISA kits. The levels of IL-1 $\beta$ and IL- 6 in the supernatant were significantly higher in the $P Q$ group compared with the control group, while the levels of these cytokines in the $P Q+C M$ group were significantly lower than those in the $P Q$ group (Fig. $2 F$ and $G$ ). The level of IL-10 in the supernatant of the control group was used as the baseline value. Compared with the control group, the other three groups exhibited significantly increased IL-10 levels, and the levels in the CM group were the highest. In addition, the IL-10 levels in the PQ + CM group were significantly higher compared with those in the PQ group (Fig. $2 \mathrm{H}$ ).

hAMSC-CM reduces PQ-induced increases in apoptosis rates. Flow cytometry was used to detect apoptosis in A549 cells. After exposure (to PQ at $750 \mu \mathrm{M}$ ) and intervention (with 50\% hAMSC-CM), apoptosis in each group was detected with an Annexin V-FITC/PI apoptosis kit. The apoptotic rate of the $\mathrm{PQ}$ group was higher than those of the other groups. The apoptosis rates of the control, $\mathrm{PQ}, \mathrm{PQ}+\mathrm{CM}$ and $\mathrm{CM}$ groups were $14.44 \pm 0.86 \%, 49.39 \pm 0.81 \%, 22.65 \pm 0.95 \%$ and $14.49 \pm 1.46 \%$, respectively. The early + late apoptotic rate of the $P Q+C M$ group was lower than that of the $P Q$ group (Fig. $3 A$ and $B$ ).

The expression levels of proteins in apoptosis-related and endoplasmic reticulum-related pathways were detected by western blot analysis. The results showed that the Bax/Bcl-2 ratio in the $\mathrm{PQ}$ group was significantly higher compared with in the control group, while the ratio in the $P Q+C M$ group was significantly lower than that in the $P Q$ group. There was no significant difference between the $P Q+C M$ group and the control group (Fig. 3C and D).

hAMSC-CM and PQ affects the protein expression level of CHOP in cells. After exposure to PQ at $750 \mu \mathrm{M}$, the protein expression of $\mathrm{CHOP}$ in the $\mathrm{PQ}$ group was increased compared with the control group, as determined by western blot analysis. The concentration of CHOP protein in the PQ group was $50 \%$ higher than that in the control group. Following hAMSC-CM treatment, the expression of CHOP protein in the PQ $+\mathrm{CM}$ group was significantly decreased compared with the $P Q$ group $(P<0.05)$ (Fig. 4A and $B)$.

\section{Discussion}


Under normal circumstances, ROS produced in the body can be quickly removed by antioxidant defense system components, such as SOD, without causing physiological damage. If ROS cannot be removed in time, they cause oxidative stress damage, resulting in cell membrane phospholipid peroxidation and inducing apoptosis. The mechanism of $P Q$ poisoning has not yet been fully elucidated, but numerous studies have confirmed that $\mathrm{PQ}$ undergoes a series of redox reactions in the body to form superoxide anions, which results in the accumulation of ROS and can directly cause cell damage $(16,17)$.

In the present study, upon observing the distribution of ROS in A549 cells, it was revealed that PQ increased ROS production in cells, resulting in cellular damage and decreased cell viability. hAMSC-CM itself did not damage A549 cells or promote cell proliferation. However, hAMSC-CM reduced the production of ROS in PQ-treated cells and alleviated PQ-induced cell damage. In addition, detection of the intermediate metabolites MDA and SOD, combined with microscopic observation of cell structure changes, revealed that hAMSC-CM reduced MDA content, increased SOD content, enhanced antioxidant capacity and alleviated cell damage caused by superoxide anions.

MSCs have certain immunosuppressive properties that can serve key roles in inflammation-related diseases. In the current experiment, IL levels were examined in A549 cell culture supernatant to evaluate the release of inflammatory cytokines, including anti- or Pro-inflamatory, after cells were exposed to PQ. It was previously revealed that hAMSC-CM contained a certain amount of IL-10, an anti-inflammatory cytokine with strong immunomodulatory activity that can inhibit inflammatory reactions. In the present study, hAMSC-CM downregulated the levels of proinflammatory factors, such as IL-1 $\beta$ and IL- 6 , and upregulated IL-10 levels, which may explain the reduction in inflammation associated with hAMSC-CM treatment $(18,19)$. It has been confirmed that Human Placental Mesenchymal Stem Cells (also termed PMSCs) can induce macrophages to stich from pro-inflammatory M1 cells (which secrete proinflammatory cytokines such as IL-1B and IL-6) into an anti-inflammatory M2 phenotype (which secretes anti-inflammatory cytokines such as IL-10). This may explain further the fact that IL-10 was upregulated, and the other two cytokines were downregulated under hAMSC-CM conditions (20).

Apoptosis, also known as programmed cell death, is primarily responsible for maintaining homeostasis by clearing abnormal or damaged cells (21). In the present study, the apoptosis rates of A549 cells were significantly increased after PQ exposure, and endoplasmic reticulum stress (ERS) and injury were significantly enhanced, suggesting that PQ exposure caused persistent or excessive ERS in cells, which resultantly induced apoptosis. Increased ERS may have been the cause of apoptosis in the A549 cells. Abnormal accumulation of apoptotic cells increases the release of inflammatory mediators, and causes a persistent inflammatory response, which in turn further enhances apoptosis and results (22). Intravenous injection of hAMSC-CM after $\mathrm{PQ}$ poisoning may reduce the production of inflammatory and damaging substances in the circulation, interrupting this cycle and ultimately reducing apoptosis. In addition, the present study revealed that hAMSC-CM intervention decreased the expression levels of Bax within cells while increasing the expression of the anti-apoptotic protein Bcl-2. 
$\mathrm{CHOP}$ is a member of the CCAAT/enhancer binding protein (C/EBP) transcription factor family, which inhibits the transcriptional activity of C/EBP and is a target for other selective enhancers (23). CHOP regulates apoptosis by activating pro-apoptotic genes and downregulating anti-apoptotic genes, such as the $\mathrm{Bcl}-2$ gene (24). Detection of the expression of CHOP protein in ERS-related pathways revealed that $\mathrm{PQ}$ exposure increased CHOP protein expression, while hAMSC-CM treatment decreased CHOP protein expression in poisoned cells, confirming that hAMSC-CM attenuates increases in ERS within PQ-exposed A549 cells. ERS can also cause apoptosis via a cell death mechanism independent of the unfolded protein response. For example, excessive ERS induces release of calcium into the cytosol, thus causing apoptosis via Bax and Bcl-2 (25).

The present in vitro cell experiment revealed that $P Q$ increases the production of ROS, which results in the release of inflammatory factors, induces oxidative stress and aggravates cell damage. hAMSC-CM alleviates the apoptosis induced by $\mathrm{PQ}$ and serves a protective role. However, the other underlying mechanisms and associated pathways still need to be further explored. hAMSC-CM has antiinflammatory and immunomodulatory effects and can attenuate PQ-induced A549 cell injury by reducing oxidative stress levels and ERS levels.

\section{Declarations}

\section{Acknowledgments}

This work was supported by the National Natural Science Foundation of China (82060346) and the Science and technology support plan of Guizhou Province [Qiankehe support plan J word (2021) General items no. 044].

\section{Availability of data and materials}

The datasets used and/or analyzed during the current study are available from the corresponding author on reasonable request.

\section{Authors' contributions}

FL and LG performed the CCK-8 experiment, apoptosis detection, ROS content detection, and Western blot analysis experiments, analyzed the data and wrote the manuscript. LJ analyzed information of transmission electron microscope pictures. LW, HT and MF performed ELISA testing experiments, and analyzed the data. $\mathrm{MZ}, \mathrm{XL}, \mathrm{YW}$ and $\mathrm{MW}$ analyzed the data and modified of the manuscript. $\mathrm{MZ}$ received funding for the project, conceived the study and performed the experiments. All authors read and approved the final manuscript.

\section{Ethics approval and consent to participate}

The experiments in this study were approved by the Experiment Ethics Committee of Zunyi Medical University (2020-1-252). 


\section{Patient consent for publication}

Not applicable.

\section{Competing interests}

The authors declare that they have no competing interests.

\section{References}

1. Gawarammana IB, Dawson AH. Peripheral burning sensation: a novel clinical marker of poor prognosis and higher plasma-paraquat concentrations in paraquat poisoning[J]. Clin Toxicol (Phila). 2010;48(4):347-9.

2. van der Wal NA, van Oirschot JF, van Dijk A, et al. Mechanism of protection of alveolar type Il cells against paraquat-induced cytotoxicity by deferoxamine[J]. Biochem Pharmacol. 1990;39(11):166571.

3. Guo F, Sun Y, Su L, et al. Losartan attenuates paraquat-induced pulmonary fibrosis in rats[J]. Human Experimental Toxicology. 2015;34(5):497-505.

4. Jianshu Huang $D$, Xuan $X$, Li, et al. The value of APACHE II in predicting mortality after paraquat poisoning in Chinese and Korean population: A systematic review and meta-analysis[J]. Med (Baltim). 2017;96(30):e6838.

5. Tirino V, Paino F, Riccardo d'Aquino, et al. Methods for the identification, characterization and banking of human DPSCs: current strategies and perspectives[J]. Stem Cell Reviews. 2011;7(3):60815.

6. Yang J-X, Zhang N, Wang H-W, et al. CXCR4 receptor overexpression in mesenchymal stem cells facilitates treatment of acute lung injury in rats[J]. J Biol Chem. 2015;290(4):1994-2006.

7. Zhang L, Wang Yu, Zhao M, et al. Combined signaling of NF-kappaB and IL-17 contributes to Mesenchymal stem cells-mediated protection for Paraquat-induced acute lung injury[J]. BMC Pulm Med. 2020;20(1):195.

8. Cetinkaya B, Unek G, Kipmen-Korgun D, et al. Effects of Human Placental Amnion Derived Mesenchymal Stem Cells on Proliferation and Apoptosis Mechanisms in Chronic Kidney Disease in the Rat[J]. International Journal of Stem Cells. 2019;30(1):151-61. 12 ).

9. Li J, Koike-Soko C, Sugimoto J, et al. Human Amnion-Derived Stem Cells Have Immunosuppressive Properties on NK Cells and Monocytes[J]. Cell Transplant. 2015;24(10):2065-76.

10. Pianta S, Bonassi Signoroni P, Muradore I, et al. Amniotic Membrane Mesenchymal Cells-Derived Factors Skew T Cell Polarization Toward Treg and Downregulate Th1 and Th17 Cells Subsets[J]. Stem Cell Reviews Reports. 2015;11(3):394-407.

11. Wu Q, Fang T, Lang $\mathrm{H}$, et al. Comparison of the proliferation, migration and angiogenic properties of human amniotic epithelial and mesenchymal stem cells and their effects on endothelial cells[J]. Int $J$ 
Mol Med. 2017;39(4):918-26.

12. Rolfo A, Giuffrida D, Giuffrida MC, et al. New perspectives for prostate cancer treatment: $\backslash$, in vitro $\backslash$, inhibition of LNCaP and PC3 cell proliferation by amnion-derived mesenchymal stromal cells conditioned media[J]. The Aging Male. 2014;17(2):94-101.

13. Yan $\mathrm{K}$, Zhang R, Chen L, et al. Nitric oxide-mediated immunosuppressive effect of human amniotic membrane-derived mesenchymal stem cells on the viability and migration of microglia[J]. Brain Res. 2014;1590:1-9.

14. Silvia Díaz-Prado, Emma Muiños-López, Tamara Hermida-Gómez, et al. Human amniotic membrane as an alternative source of stem cells for regenerative medicine[J]. Differentiation. 2011; 81(3): $162-$ 71.

15. Yaxin L. Duo Wang. Inhibition of Colon Cancer Cell Growth by Imidazole Through Activation of Apoptotic Pathway[J]. Med Sci Monit. 2019;25:7597-604.

16. Huang CL, Chao CC, Lee YC, et al. Paraquat Induces Cell Death Through Impairing Mitochondrial Membrane Permeability[J]. Mol Neurobiol. 2016;53(4):2169-88.

17. Chang X, Lu W, Dou T, et al. Paraquat inhibits cell viability via enhanced oxidative stress and apoptosis in human neural progenitor cells[J]. Chem Biol Interact. 2013;206(2):248-55.

18. Pedrazza L, Lunardelli A, Luft C, et al. Mesenchymal stem cells decrease splenocytes apoptosis in a sepsis experimental model[J]. Inflamm Res. 2014;63(9):719-28.

19. Pedrazza L, Cubillos-Rojas M, De Mesquita FC, et al. Mesenchymal stem cells decrease lung inflammation during sepsis, acting through inhibition of the MAPK pathway[J]. Stem Cell Res Ther. 2017;8(1):289.

20. Abumaree MH, Al Jumah MA, Kalionis B, et al. Human Placental Mesenchymal Stem Cells (pMSCs) Play a Role as Immune Suppressive Cells by Shifting Macrophage Differentiation From Inflammatory M1 to Anti-Inflammatory M2 Macrophages. Stem Cell Rev Rep. 2013;9(5):620-41.

21. Sauler M, Bazan IS, Lee PJ. Cell Death in the Lung: The Apoptosis-Necroptosis Axis[J]. Annu Rev Physiol. 2019;81:375-402.

22. Robb CT, Regan KH, Dorward DA, et al. Key mechanisms governing resolution of lung inflammation[J]. Seminars in Immunopathology. 2016;38(4):425-48.

23. Cassel TN, Nord M. C/EBP transcription factors in the lung epithelium[J]. American Journal of Physiology-Lung Cellular Molecular Physiology. 2003;285(4):L773-81.

24. Mccullough KD, Martindale JL, Klotz LO, et al. Gadd153 Sensitizes Cells to Endoplasmic Reticulum Stress by Down-Regulating Bcl2 and Perturbing the Cellular Redox State[J]. Mol Cell Biol. 2001;21(4):1249-59.

25. Scorrano L, Oakes SA, Opferman JT, et al. BAX and BAK Regulation of Endoplasmic Reticulum Ca2+: A Control Point for Apoptosis[J]. Science. 2003;300(5616):135-9.

\section{Figures}



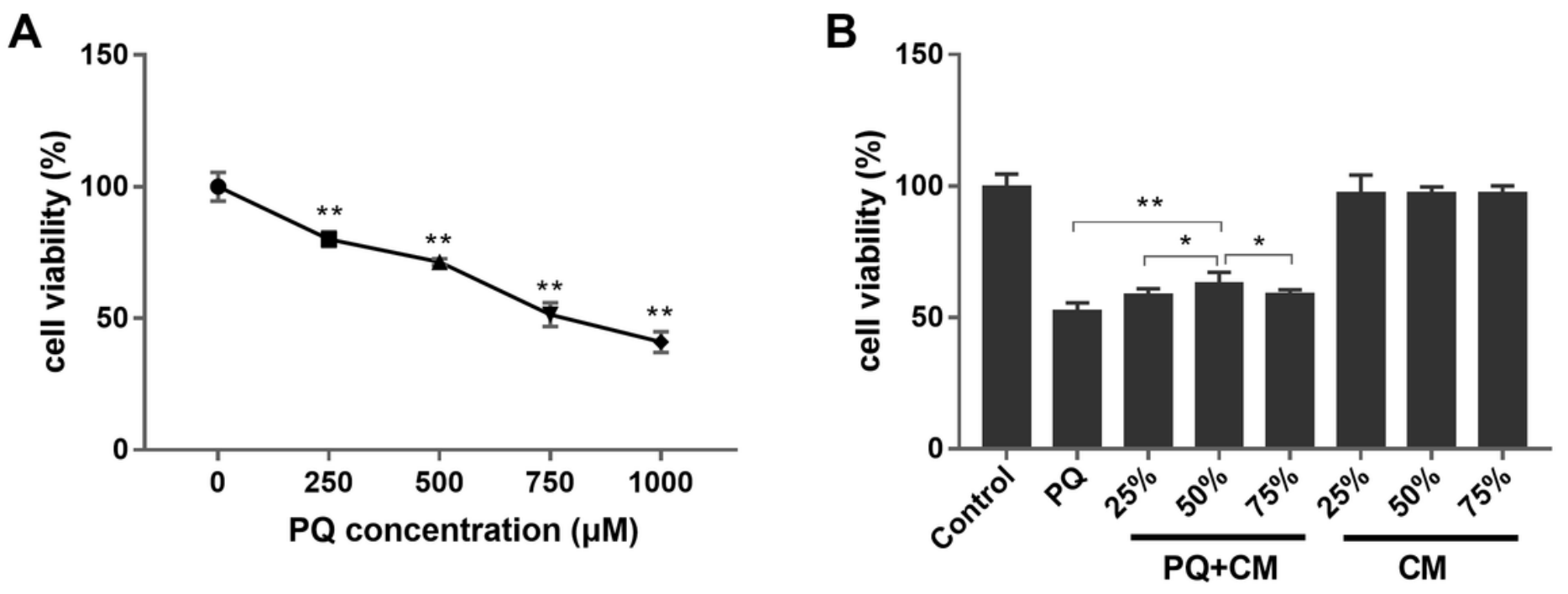

\section{Figure 1}

$\mathrm{PQ}$ reduces the proliferation of $\mathrm{A} 549$ cells and hAMSC-CM attenuates this reduction. (A) Comparison of the effects of exposure to different concentrations of PQ (250,500, 750 and 1,000 $\mu \mathrm{M})$ and the control condition $(0 \mu \mathrm{M})$ on cell viability using CCK-8. ${ }^{*} \mathrm{P}<0.01$ vs. control group. (B) Cell viability after treatment with $\mathrm{PQ}$ and various concentrations of hAMSC-CM was determined using CCK-8. $n=6 .{ }^{*} P<0.05 ; * * P<0.01$ as indicated. $\mathrm{PQ}$, paraquat; hAMSC, human amnion-derived mesenchymal stem cells; $\mathrm{CM}$, conditioned medium; CCK-8, Cell Counting Kit-8. 

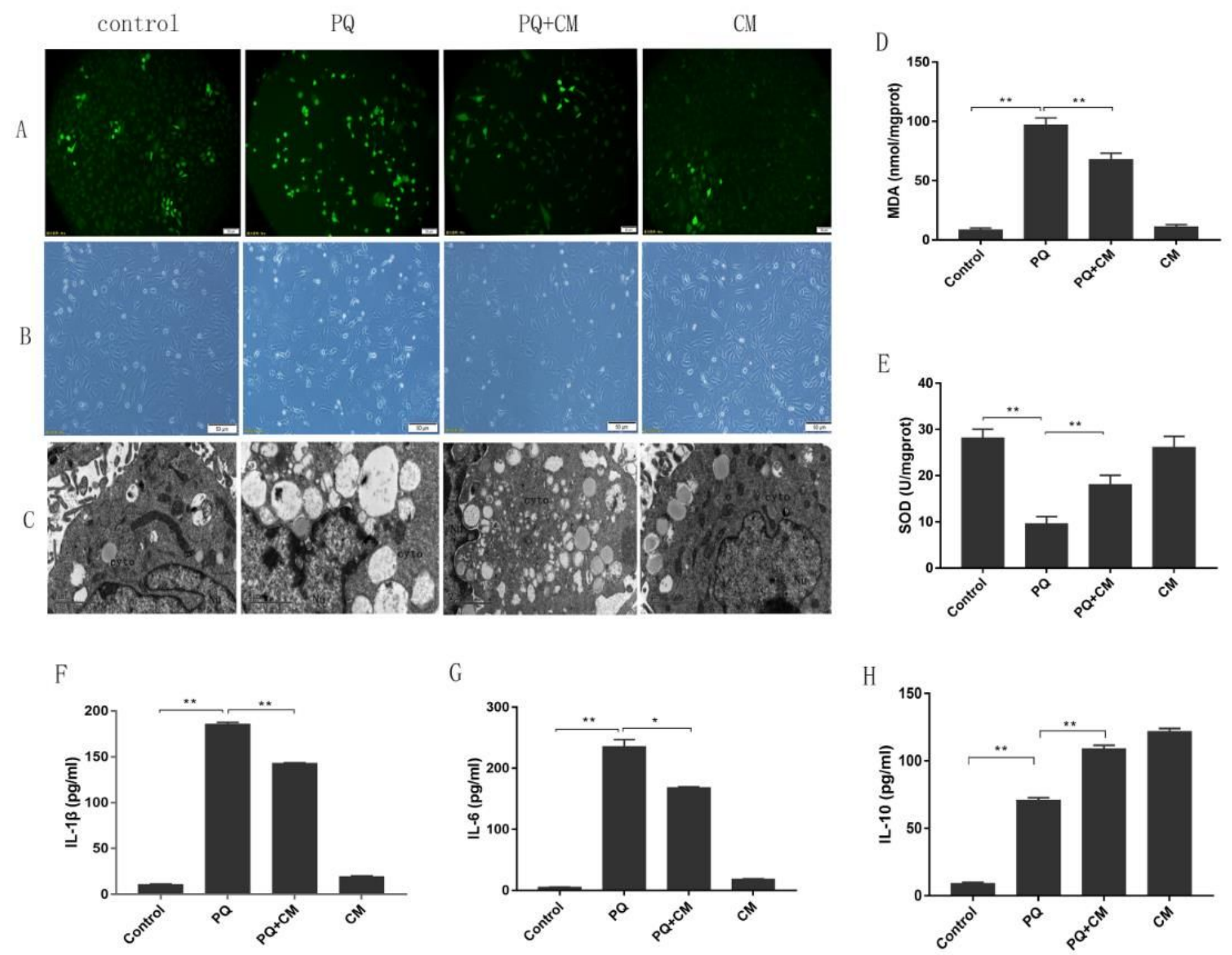

\section{Figure 2}

hAMSC-CM reduces PQ-induced oxidative stress and alleviates increases in inflammatory mediator levels in $A 549$ cells. ${ }^{*} \mathrm{P}<0.05 ;{ }^{*} \mathrm{P}<0.01$ as indicated. $\mathrm{PQ}$, paraquat; $\mathrm{hAMSC}$, human amnion-derived mesenchymal stem cells; CM, conditioned medium; SOD, superoxide dismutase; Nu, nucleus; cyto, cytoplasm. 

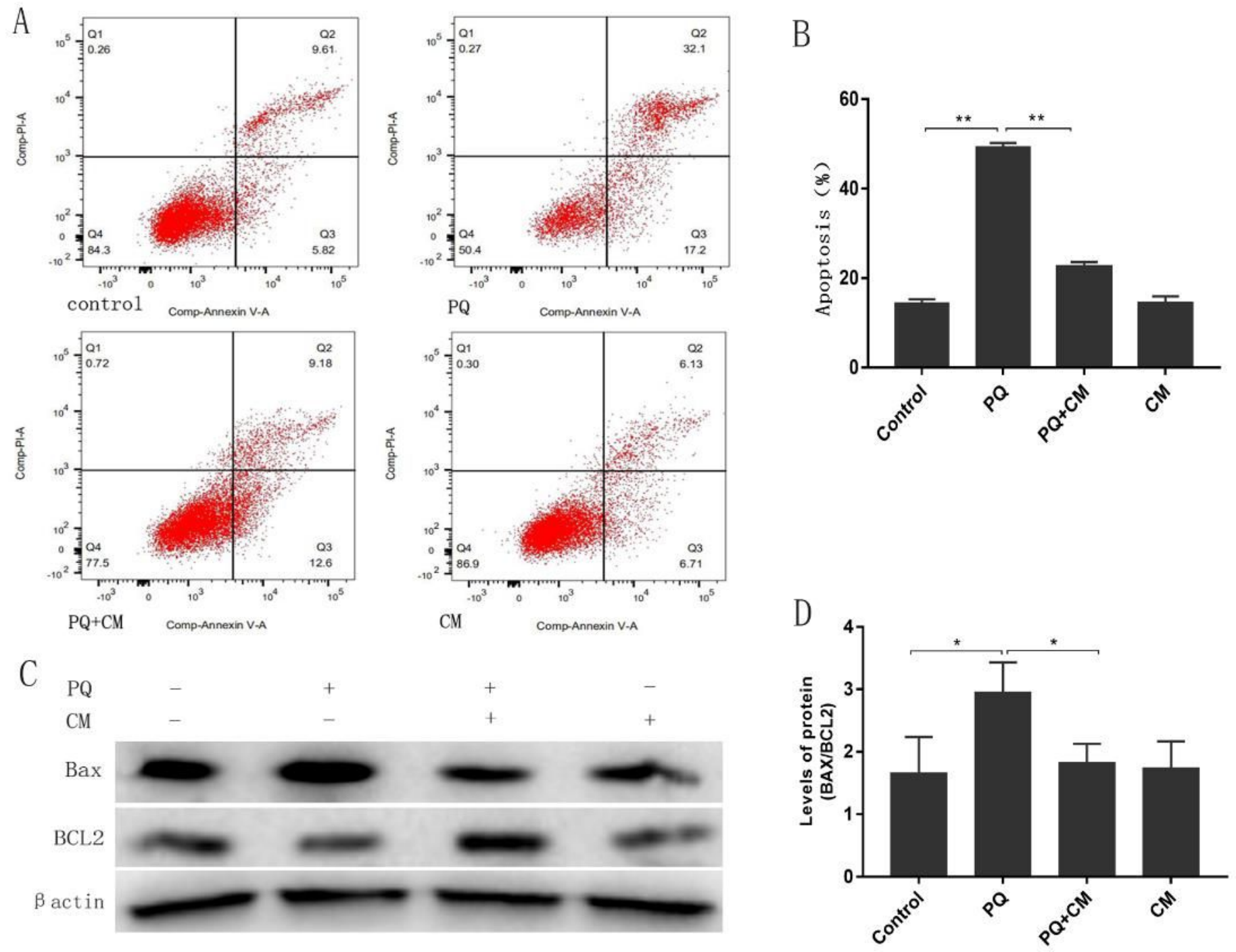

Figure 3

hAMSC-CM reduces PQ-induced apoptosis in $A 549$ cells. ${ }^{*} \mathrm{P}<0.05$; ${ }^{\star *} \mathrm{P}<0.01$ as indicated. $\mathrm{PQ}$, paraquat; hAMSC, human amnion-derived mesenchymal stem cells; $\mathrm{CM}$, conditioned medium.
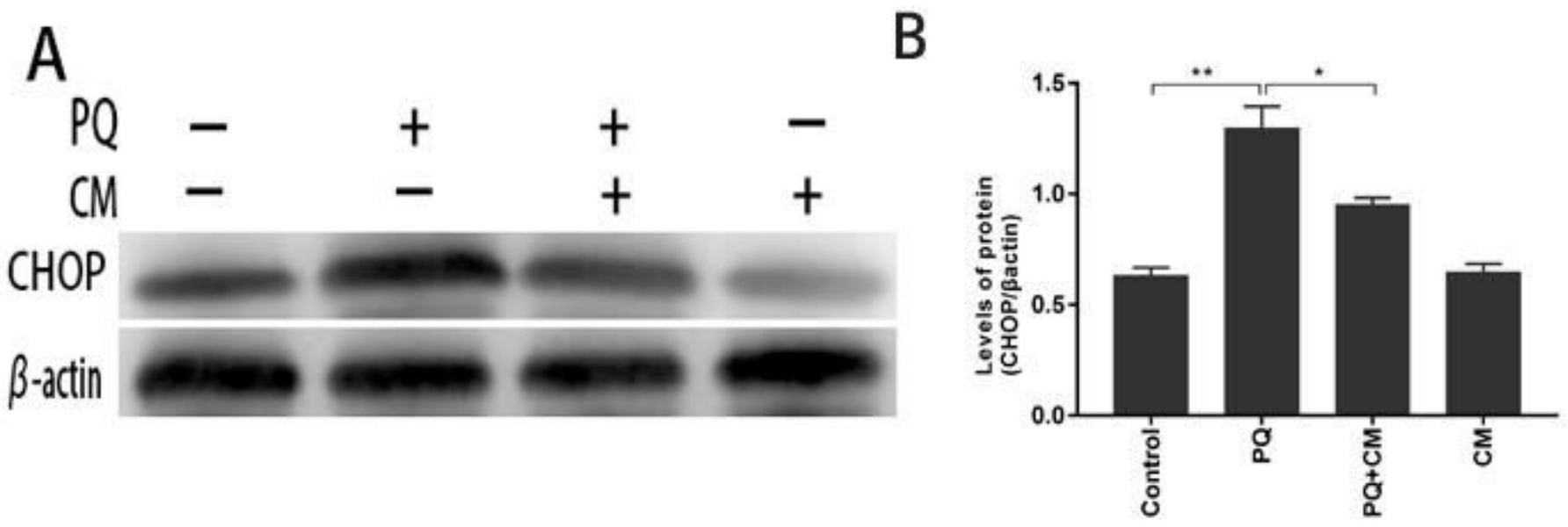
Figure 4

Western blot analysis of intracellular CHOP protein expression. ${ }^{*}<<0.05 ;{ }^{*} \mathrm{P}<0.01$ as indicated. $\mathrm{PQ}$, paraquat; hAMSC, human amnion-derived mesenchymal stem cells; CM, conditioned medium. 